\title{
Application of the Subordination Principle to the Harmonic Mappings Convex in One Direction with Shear Construction Method
}

\author{
Yaşar Polatoğlu, H. Esra Özkan, and Emel Yavuz Duman \\ Department of Mathematics and Computer Science, İstanbul Kültür University, İstanbul 31456, Turkey \\ Correspondence should be addressed to H. Esra Özkan, e.ozkan@iku.edu.tr
}

Received 3 June 2010; Accepted 26 July 2010

Academic Editor: N. Govil

Copyright (C) 2010 Yaşar Polatoğlu et al. This is an open access article distributed under the Creative Commons Attribution License, which permits unrestricted use, distribution, and reproduction in any medium, provided the original work is properly cited.

Any harmonic function in the open unit disc $\mathbb{D}=\{z|| z \mid<1\}$ can be written as a sum of an analytic and antianalytic functions $f=h(z)+\overline{g(z)}$, where $h(z)$ and $g(z)$ are analytic functions in $\mathbb{D}$ and are called the analytic part and the coanalytic part of $f$, respectively. Many important questions in the study of the classes of functions are related to bounds on the modulus of functions (growth) or the modulus of the derivative (distortion). In this paper, we consider both of these questions.

\section{Introduction}

Let $U$ be a simply connected domain in the complex plane. A harmonic function $f$ has the representation $f=h(z)+\overline{g(z)}$, where $h(z)$ and $g(z)$ are analytic in $U$ and are called the analytic and coanalytic parts of $f$, respectively. Let $h(z)=a_{0}+a_{1} z+a_{2} z^{2}+\cdots$, and $g(z)=$ $b_{0}+b_{1} z+b_{2} z^{2}+\cdots$ be analytic functions in the open unit disc $\mathbb{D}$. If $J_{f}(z)=\left|h^{\prime}(z)\right|^{2}-\left|g^{\prime}(z)\right|^{2}>0$, then $f=h(z)+\overline{g(z)}$ is called the sense-preserving harmonic univalent function in $\mathbb{D}$. The class of all sense-preserving harmonic univalent functions is denoted by $\mathcal{S}_{H}$, with $a_{0}=b_{0}=0$, $a_{1}=1$, and $\left|b_{1}\right|<1$, and the class of all sense-preserving harmonic univalent functions is denoted by $\mathcal{S}_{H}^{0}$ with $a_{0}=b_{0}=b_{1}=0, a_{1}=1$. For convenience, we will examine sensepreserving functions, that is, functions for which $J_{f}(z)>0$. If $f$ has $J_{f}(z)<0$, then $\bar{f}$ is sense preserving. The analytic dilatation of the harmonic functions is given by $w(z)=g^{\prime}(z) / h^{\prime}(z)$. We also note that if $f$ is locally univalent and sense preserving then $|w(z)|<1$.

In this paper we examine the class of functions that are convex in one direction. The shear construction is essential to the present work as it allows one to study harmonic functions through their related analytic functions as shown in [1] by Hengartner and Schober. 
The shear construction produces a univalent harmonic function that maps $\mathbb{D}$ to the region that is convex in the direction of the real axis. This construction relies on the following theorem of Clunie and Sheil-Small.

Theorem 1.1 (see [2]). A harmonic function $f=h(z)+\overline{g(z)}$ locally univalent in $\mathbb{D}$ is a univalent mapping of $\mathbb{D}$ onto a domain convex in the direction of the real axis if and only if $(h(z)-g(z))$ is a conformal univalent mapping of $\mathbb{D}$ onto a domain convex in the direction of the real axis.

Theorem 1.1 leads to the construction of univalent harmonic function with analytic dilatation $w(z)$. Hengartner and Schober [1] studied the analytic functions $\psi(z)$ that are convex in the direction of the imaginary axis. They used a normalization which requires, in essence, that right and left extremes of $\psi(\mathbb{D})$ be the image of $(+1)$ and $(-1)$. This normalization is that there exist points $\left(z_{n}^{\prime}\right)$ converging to $z=1$ and $z_{n}^{\prime \prime}$ converging to $z=-1$ such that

$$
\begin{aligned}
& \lim _{n \rightarrow \infty}\left\{\operatorname{Re} \psi\left(z_{n}^{\prime}\right)\right\}=\operatorname{Sup}_{|z|<1}\{\operatorname{Re} \psi(z)\}, \\
& \lim _{n \rightarrow \infty}\left\{\operatorname{Re} \psi\left(z_{n}^{\prime \prime}\right)\right\}=\operatorname{Inf}_{|z|<1}\{\operatorname{Re} \psi(z)\} .
\end{aligned}
$$

If CIA is the class of domains, $\mathbb{D}$, that are convex in the direction of the imaginary axis and that admit a mapping $\psi(z)$ so that $\psi(\mathbb{D})=\mathbb{D}$ and satisfies the normalization (1.1), then we have the following result.

Theorem 1.2 (see [1]). Suppose that $\psi(z)$ is analytic and nonconstant for $|z|<1$, then one has $\operatorname{Re}\left[\left(1-z^{2}\right) \psi^{\prime}(z)\right]>0$ if and only if

(i) $\psi(z)$ is univalent on $\mathbb{D}$,

(ii) $\psi(z) \in \subset I A$,

(iii) $\psi(z)$ is normalized by (1.1).

Using this characterization of functions, Hengartner and Schober proved the following theorem.

Theorem 1.3 (see [1]). If $\psi(z)$ is analytic for $|z|<1$ and satisfies $\operatorname{Re}\left[\left(1-z^{2}\right) \psi^{\prime}(z)\right] \geq 0$, then

$$
\frac{(1-r)\left|\psi^{\prime}(0)\right|}{(1+r)(1+r)^{2}} \leq \frac{\left|\psi^{\prime}(0)\right|}{(1-r)^{2}} .
$$

To be able to obtain this result for functions that are in the direction of the real axis, let us consider the following situation. Suppose that $\varphi(z)$ is a function that is analytic and convex in the direction of the real axis. Furthermore, suppose that $\varphi(z)$ is normalized by the following.

Let there exist points $\left(z_{n}^{\prime}\right)$ converging to $z=e^{i \alpha}$ and $\left(z_{n}^{\prime \prime}\right)$ converging to $z=e^{i(\alpha+\pi)}$, such that

$$
\begin{aligned}
& \lim _{n \rightarrow \infty}\left\{\operatorname{Im} \varphi\left(z_{n}^{\prime}\right)\right\}=\operatorname{Sup}_{|z|<1}\{\operatorname{Im} \varphi(z)\}, \\
& \lim _{n \rightarrow \infty}\left\{\operatorname{Re} \varphi\left(z_{n}^{\prime \prime}\right)\right\}=\operatorname{Inf}_{|z|<1}\{\operatorname{Im} \varphi(z)\} .
\end{aligned}
$$


Consequently, if $\psi(z)$ satisfies (1.1), then $\varphi(z)=i \psi(z)\left(e^{-i \alpha} z\right)$ satisfies (1.3). Knowing this, we can apply $\varphi(z)$ and see that the result still holds, with $\psi(z)$ being replaced by $\varphi(z)$. In this situation, $\operatorname{Re}\left[(-i)\left(e^{i \alpha}-e^{-i \alpha} z^{2}\right) \varphi^{\prime}(z)\right]>0$. We can now prove the derivative bounds for the harmonic function convex in the direction of the real axis.

Finally, let $\Omega$ be the family of functions $\phi(z)$ which are analytic in $\mathbb{D}$ and satisfying the condition $\phi(0)=0,|\phi(z)|<1$ for every $z \in \mathbb{D}$. Denote by $p$ the class of analytic functions $p(z)$ given by $p(z)=1+p_{1} z+p_{z} z^{2}+\cdots$ which satisfy $\operatorname{Re} p(z)>0$ for all $z \in \mathbb{D}$. Let $s_{1}(z)=z+c_{2} z^{2}+\cdots$ and $s_{2}(z)=z+d_{2} z^{2}+\cdots$ be analytic functions in $\mathbb{D}$. If $s_{1}(z)=s_{2}(\phi(z))$ is satisfied for some $\phi(z) \in \Omega$ and every $z \in \mathbb{D}$, then we say that $s_{1}(z)$ is subordinate to $s_{2}(z)$, and we write $s_{1}(z) \prec s_{2}(z)$.

\section{Main Results}

Lemma 2.1. Let $f=h(z)+\overline{g(z)}$ be an element of $\mathcal{S}_{H}$, and let $w(z)=g^{\prime}(z) / h^{\prime}(z)$ be the analytic dilatation of $f$, then

$$
\begin{gathered}
\frac{\left|b_{1}\right|-r}{1-\left|b_{1}\right| r} \leq|w(z)| \leq \frac{\left|b_{1}\right|+r}{1+\left|b_{1}\right| r}, \\
\frac{\left(1-\left|b_{1}\right|\right)(1-r)}{1+\left|b_{1}\right| r} \leq(1-|w(z)|) \leq \frac{\left(1-\left|b_{1}\right|\right)(1+r)}{1-\left|b_{1}\right| r}, \\
\frac{\left(1+\left|b_{1}\right|\right)(1-r)}{1-\left|b_{1}\right| r} \leq(1+|w(z)|) \leq \frac{\left(1+\left|b_{1}\right|\right)(1+r)}{1+\left|b_{1}\right| r}, \\
\frac{(1-r)^{2}\left(1-\left|b_{1}\right|^{2}\right)}{1-\left|b_{1}\right|^{2} r^{2}} \leq 1-|w(z)|^{2} \leq \frac{\left(1-r^{2}\right)\left(1-\left|b_{1}\right|^{2}\right)}{\left(1-\left|b_{1}\right| r\right)^{2}} .
\end{gathered}
$$

Proof. Since $f=h(z)+\overline{g(z)} \in \mathcal{S}_{H}$, then

$$
w(z)=\frac{\left(b_{1} z+b_{2} z^{2}+\cdots\right)^{\prime}}{\left(z+a_{2} z^{2}+\cdots\right)^{\prime}}=\frac{b_{1}+2 b_{2} z+\cdots}{1+a_{2} z+\cdots} \Longrightarrow w(0)=b_{1}
$$

Now, we define the function

$$
\phi(z)=\frac{w(z)-w(0)}{1-\overline{w(0)} w(z)}=\frac{w(z)-b_{1}}{1-\overline{b_{1}} w(z)}
$$

This function satisfies the conditions of the Schwarz lemma. Then, we have

$$
w(z)=\frac{\phi(z)+b_{1}}{1+\overline{b_{1}} \phi(z)}
$$

Using the principle of subordination and (2.7), we see that the analytic dilatation $w(z)$ is subordinate to $\left(\left(z+b_{1}\right) /\left(1+\overline{b_{1}} z\right)\right)$. On the other hand, the transformation $\left(\left(z+b_{1}\right) /\left(1+\overline{b_{1}} z\right)\right)$ 
maps $|z|=r$ onto the circle with the centre $C(r)=\left(\alpha_{1}\left(1-r^{2}\right) /\left(1-\left|b_{1}\right|^{2} r^{2}\right), \alpha_{2}\left(1-r^{2}\right) /\left(1-\left|b_{1}\right|^{2} r^{2}\right)\right)$ and the radius $\rho(r)=\left(1-\left|b_{1}\right|^{2}\right) r /\left(1-\left|b_{1}\right|^{2} r^{2}\right)$, where $b_{1}=\alpha_{1}+i \alpha_{2}$. Thus, again using the subordination principle, we write

$$
\left|w(z)-\frac{b_{1}\left(1-r^{2}\right)}{1-\left|b_{1}\right|^{2} r^{2}}\right| \leq \frac{\left(1-\left|b_{1}\right|^{2}\right) r}{1-\left|b_{1}\right|^{2} r^{2}} .
$$

Following some simple calculations from (2.8), we get (2.1), (2.2), (2.3), and (2.4).

Theorem 2.2. Let $f=h(z)+\overline{g(z)}$ be an element of $\mathcal{S}_{H}$, and let $f$ be convex in the direction of the real axis, and let $\varphi(z)=h(z)-g(z), w(z)=g^{\prime}(z) / h^{\prime}(z)$. Furthermore, let $\varphi(z)$ satisfy the normalization (1.1), then for $|z|<1$, one has

$$
\begin{gathered}
\frac{\left|1-b_{1}\right|\left(1+\left|b_{1}\right| r\right)(1-r)}{\left(1+\left|b_{1}\right|\right)(1+r)^{2}\left(1+r^{2}\right)} \leq\left|f_{z}\right| \leq \frac{\left|1-b_{1}\right|\left(1+\left|b_{1}\right| r\right)}{\left(1-\left|b_{1}\right|\right)(1-r)^{3}}, \\
\frac{|w(z)|\left|1-b_{1}\right|(1-r)\left(1+\left|b_{1}\right| r\right)}{\left(1+\left|b_{1}\right|\right)(1+r)^{2}\left(1+r^{2}\right)} \leq\left|f_{\bar{z}}\right| \leq \frac{\left|1-b_{1}\right|\left(1+\left|b_{1}\right| r\right) r}{\left(1-\left|b_{1}\right|\right)(1-r)^{3}} .
\end{gathered}
$$

Proof. Since $\varphi(z)=h(z)-g(z) \Rightarrow \varphi^{\prime}(z)=h^{\prime}(z)-g^{\prime}(z), g^{\prime}(z)=h^{\prime}(z) w(z)$, then we have

$$
\begin{aligned}
& f_{z}=h^{\prime}(z)=\frac{\varphi^{\prime}(z)}{1-w(z)} \\
& \overline{f_{\bar{z}}}=g^{\prime}(z)=\frac{w(z) \varphi^{\prime}(z)}{1-w(z)} .
\end{aligned}
$$

Since analytic dilatation $w(z)$ satisfies the condition $|w(z)|<1$ for every $z \in \mathbb{D}$, then we have

$$
\begin{gathered}
\frac{\left|\varphi^{\prime}(z)\right|}{1+|w(z)|} \leq\left|f_{z}\right| \leq \frac{\left|\varphi^{\prime}(z)\right|}{1-|w(z)|^{\prime}} \\
\frac{|w(z)|\left|\varphi^{\prime}(z)\right|}{1+|w(z)|} \leq\left|\overline{f_{\bar{z}}}\right| \leq \frac{|w(z)|\left|\varphi^{\prime}(z)\right|}{1-|w(z)|} .
\end{gathered}
$$

Using (2.2), (2.3), and (1.2) in (2.11), we get

$$
\begin{gathered}
\frac{(1-r)\left(1+\left|b_{1}\right| r\right)\left|\varphi^{\prime}(0)\right|}{\left(1+\left|b_{1}\right|\right)(1+r)^{2}\left(1+r^{2}\right)} \leq\left|f_{z}\right| \leq \frac{\left(1+\left|b_{1}\right| r\right)\left|\varphi^{\prime}(0)\right|}{\left(1-\left|b_{1}\right|\right)(1-r)^{3}}, \\
\frac{|w(z)|(1-r)\left(1+\left|b_{1}\right| r\right)\left|\varphi^{\prime}(0)\right|}{\left(1+\left|b_{1}\right|\right)(1+r)^{2}\left(1+r^{2}\right)} \leq\left|f_{\bar{z}}\right| \leq \frac{\left(1+\left|b_{1}\right| r\right)\left|\varphi^{\prime}(0)\right| r}{\left(1-\left|b_{1}\right|\right)(1-r)^{3}} .
\end{gathered}
$$

On the other hand, $\varphi(z)=h(z)-g(z) \Rightarrow \varphi^{\prime}(z)=h^{\prime}(z)-g^{\prime}(z) \Rightarrow \varphi^{\prime}(0)=1-b_{1}$ therefore, (2.12) can be written in the form (2.9). 
Corollary 2.3. If one lets $b_{1}=0$, then $\varphi^{\prime}(0)=1$ therefore, one obtains

$$
\begin{aligned}
& \frac{1-r}{(1+r)^{2}\left(1+r^{2}\right)} \leq\left|f_{z}\right| \leq \frac{1}{(1-r)^{3}}, \\
& \frac{|w(z)|(1-r)}{(1+r)^{2}\left(1+r^{2}\right)} \leq\left|f_{\bar{z}}\right| \leq \frac{r}{(1-r)^{3}} .
\end{aligned}
$$

These distortions were found by Schaubroeck [3].

Theorem 2.4. Let $f=h(z)+\overline{g(z)}$ be convex in the direction of the real axis, let $f=h(z)+\overline{g(z)} \in \mathcal{S}_{H}$, and let $\varphi(z)=h(z)-g(z)$ satisfy the normalization (1.1). Then, for $|z|<r$, one has

$$
|f| \leq \frac{\left|1-b_{1}\right|}{1-\left|b_{1}\right|}\left[\int_{0}^{r} \frac{\left(1+\left|b_{1}\right| \rho\right)(1+\rho)}{(1-\rho)^{3}} d \rho\right] .
$$

Proof. Since $f=h(z)+\overline{g(z)}$, we have the following inequalities:

$$
\begin{aligned}
f & =h(z)+\overline{g(z)}=\int_{0}^{r} h^{\prime}\left(\rho e^{i \theta}\right) e^{i \theta} d \rho+\overline{\int_{0}^{r} g^{\prime}\left(\rho e^{i \theta} e^{i \theta} d \rho\right)} \\
& =\int_{0}^{r} f_{z}\left(\rho e^{i \theta}\right) e^{i \theta} d \rho+\overline{\int_{0}^{r} f_{\bar{z}}\left(\rho e^{i \theta}\right) e^{-i \theta} d \rho .}
\end{aligned}
$$

Hence,

$$
|f|=|h(z)+\overline{g(z)}| \leq|h(z)|+|g(z)| \leq \int_{0}^{r}\left|f_{z}\left(\rho e^{i \theta}\right)\right| d \rho+\overline{\int_{0}^{r}\left|f_{\bar{z}}\left(\rho e^{i \theta}\right)\right| d \rho} .
$$

Applying (2.9) to the above expression yields (2.14).

Corollary 2.5. If one takes $b_{1}=0$, then one obtains

$$
|f| \leq \frac{r}{(1-r)^{2}}
$$

This growth was found by Schaubroeck [3].

Theorem 2.6. Let $f=(h(z)+g(z)) \in \mathcal{S}_{H}$, and let $f$ be convex in the direction of the real axis. If $\varphi(z)=h(z)-g(z)$ satisfies the normalization (1.1), then

$$
\frac{\left(1-\left|b_{1}\right|\right)\left(1+\left|b_{1}\right| r\right)(1-r)^{4}\left|1-b_{1}\right|^{2}}{\left(1+\left|b_{1}\right|\right)\left(1-\left|b_{1}\right| r\right)(1+r)^{4}\left(1+r^{2}\right)^{2}} \leq J_{f}(z) \leq \frac{\left(1+\left|b_{1}\right|\right)\left(1+\left|b_{1}\right| r\right)(1+r)\left|1-b_{1}\right|^{2}}{\left(1-\left|b_{1}\right|\right)\left(1-\left|b_{1}\right| r\right)(1-r)^{5}}
$$

Proof. Since $J_{f}(z)=\left|h^{\prime}(z)\right|^{2}-\left|g^{\prime}(z)\right|^{2}=\left|h^{\prime}(z)\right|^{2}\left(1-|w(z)|^{2}\right)$, then using Lemma 2.1 and Theorem 2.2 and after straightforward calculations, we get (2.18). 
Remark 2.7. We note that the distortion and growth theorem in our study is sharp, because by choosing the suitable analytic dilatation and $\varphi(z)$, we can find the extremal function in the following manner:

$$
\begin{aligned}
& \varphi(z)=h(z)-g(z) \Longrightarrow \varphi^{\prime}(z)=h^{\prime}(z)-g^{\prime}(z) \\
& w(z)=\frac{g^{\prime}(z)}{h^{\prime}(z)} \Longrightarrow 0=w(z) h^{\prime}(z)-g^{\prime}(z) \\
& h^{\prime}(z)=f_{z}=\frac{\varphi^{\prime}(z)}{1-w(z)} \Longrightarrow h(z)=\int_{0}^{z} \frac{\varphi^{\prime}(\xi)}{1-w(\xi)} d \xi \\
& g^{\prime}(z)=\overline{f_{\bar{z}}}=\frac{\varphi^{\prime}(z) w(z)}{1-w(z)} \Longrightarrow \\
& g(z)=\int_{0}^{z} \frac{\varphi^{\prime}(\xi) w(\xi)}{1-w(\xi)} d \xi=\int_{0}^{z} \frac{\varphi^{\prime}(\xi) w(\xi)-\varphi^{\prime}(\xi)+\varphi^{\prime}(\xi)}{1-w(\xi)} d \xi=\int_{0}^{z}\left[\frac{\varphi^{\prime}(\xi)}{1-w(\xi)}-\varphi^{\prime}(\xi)\right] d \xi \Longrightarrow \\
& g(z)=\int_{0}^{z} \frac{\varphi^{\prime}(\xi)}{1-w(\xi)} d \xi-\int_{0}^{z} \varphi^{\prime}(\xi) d \xi=\int_{0}^{z} \frac{\varphi^{\prime}(\xi)}{1-w(\xi)} d \xi-\varphi(z) .
\end{aligned}
$$

Therefore we have

$$
\begin{aligned}
f=h(z)+\overline{g(z)} & =\int_{0}^{z} \frac{\varphi^{\prime}(\xi)}{1-w(\xi)} d \xi+\overline{\int_{0}^{z} \frac{\varphi^{\prime}(\xi)}{1-w(\xi)} d \xi-\varphi(z)} \\
& =\int_{0}^{z} \frac{\varphi^{\prime}(\xi)}{1-w(\xi)} d \xi+\overline{\int_{0}^{z} \frac{\varphi^{\prime}(\xi)}{1-w(\xi)} d \xi}-\overline{\varphi(z)} \Longrightarrow \\
f(z) & =\operatorname{Re}\left(\int_{0}^{z} \frac{2 \varphi^{\prime}(\xi)}{1-w(\xi)} d \xi\right)-\overline{\varphi(z)}
\end{aligned}
$$

\section{References}

[1] W. Hengartner and G. Schober, "On Schlicht mappings to domains convex in one direction," Commentarii Mathematici Helvetici, vol. 45, pp. 303-314, 1970.

[2] J. Clunie and T. Sheil-Small, "Harmonic univalent functions," Annales Academiae Scientiarum Fennicae. Series A I. Mathematica, vol. 9, pp. 3-25, 1984.

[3] L. E. Schaubroeck, "Growth, distortion and coefficient bounds for plane harmonic mappings convex in one direction," The Rocky Mountain Journal of Mathematics, vol. 31, no. 2, pp. 625-639, 2001. 